\title{
Influence of JAK2V617F allele burden on clinical phenotype of polycythemia vera patients: A study from India
}

\author{
Sudha Sazawal, Kanwaljeet Singh, Sunita Chhikara, Rekha Chaubey, Manoranjan Mahapatra, Renu Saxena
}

\begin{abstract}
Background: Elevated JAK2V6I7F allele burden is associated with enhanced expression of downstream target genes in Philadelphia negative chronic myeloproliferative neoplasms (CMPNs) which include PV, ET \& PMF. Previous studies have shown the impact of JAK2V6I7F allele burden on clinical phenotype of CMPNs. However, there is no data from India regarding the association between JAK2V6I7F allele burden and clinical phenotype in PV. Aims/Settings and Design: We aimed to investigate the effect of allele burden on clinical phenotype in 90 JAK2V6I7F positive PV patients and to see its influence on disease related complications. Material and Methods: Allele burden of 90 JAK2V6I7F positive PV patients was quantified by Real-time polymerase chain reaction (RQ-PCR). Results: 74/90 (82.22\%) were males and I6/90 (17.78\%) were females (median 45 years, range 35-78). Patients with age $>50$ years had significantly higher JAK2V6I7F allele burden (median $40.15 \%$, range $0.49-91.62 \%$ ) than patients with $\leq 50$ years age (median $48.59 \%$, range $0.56-86.74 \% ; P<0.032$ ). Patients with splenomegaly had significantly higher JAK2V6I7F allele burden (mean $50.24 \%$, range $6.91-84.17 \%$ ) than patients without splenomegaly (mean $33.82 \%$, range $0.49-71.83 \% ; P<0.017$ ). Patients with higher allele burden (median 57.20 , range 43.4-72.03\%) had significantly raised thrombotic events than the patients with lower allele burden (median 37.38, range $0.49-84.17 \% ; P<0.043$ ). $49 / 90$ (54\%) were homozygous and $4 \mathrm{I} / 90$ (46\%) were heterozygous. Conclusions: Higher JAK2V6I7F allele burden showed association with increased age, splenomegaly and thrombotic events. Thus, it may be considered for prognostication and setting up the treatment protocol in PV patients.
\end{abstract}

Key words: JAK2V6I7F allele burden, polycythemia vera, real-time polymerase chain reaction

\section{Introduction}

Philadelphia chromosome-negative chronic myeloproliferative neoplasms (CMPNs) are clonal hematopoietic disorders which include polycythemia vera (PV), primary myelofibrosis (PMF), and essential thrombocythemia (ET). A gain-of-function point mutation has been reported in the Janus 2 tyrosine kinase (JAK2) gene which increases the activity of JAK2 kinase and is known to affect the clinical outcome. ${ }^{[1,2]}$ The JAK2V617F mutation is reported in approximately $90 \%-95 \%$ of $\mathrm{PV}$ patients and in approximately $50 \%-60 \%$ of ET and PMF patients. $^{[3,4]}$

Mitotic recombination leads to homozygosity in JAK2V617F mutation and is present in about one-third of PV patients. ${ }^{[2-4]}$ Previous studies have analyzed the impact of JAK2V617F mutation burden on disease phenotype by taking $50 \%$ as the cutoff value since homozygosity of JAK2V617F mutation was seen in patients with $>50 \%$ allele burden. ${ }^{[5,6]}$ However, due to the presence of mixed population of wild-type cells in the total cells harboring both homozygous and heterozygous mutation, the actual burden of homozygous JAK2V617F mutation can be under 50\%.

Elevated JAK2V617F allele burden is associated with enhanced expression of downstream target genes and increased granulocyte activation. ${ }^{[5,7-10]}$ Various studies have shown association between the allele burden and clinical phenotype. ${ }^{[1-13]}$ The impact of JAK2V617F allele burden on several clinical parameters such as hemoglobin concentration, white blood cell (WBC) counts, platelet counts, and spleen size has been reported in CMPN patients. ${ }^{[11,12]}$ In addition, the association between JAK2V617F allele burden and thrombotic risk has also been reported. ${ }^{[14,15]}$

Studies from India have reported the prevalence of JAK2 V617F mutation in CMPNs and correlated it with the clinicohematologic characteristics. ${ }^{[16,17]}$ However, there is no data

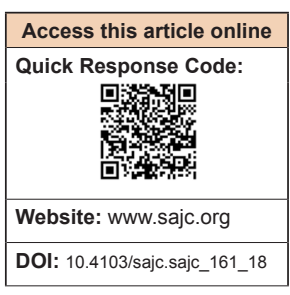

Department of Hematology, All India Institute of Medical Sciences, New Delhi, India Correspondence to: Dr. Sudha Sazawal, E-mail: sudha_sazawal@hotmail.com regarding the association between JAK2V617F allele burden and clinical phenotype in PV.

In view of this, we sought to look for the effect of JAK2V617F allele burden on clinical phenotype in a study group of ninety JAK2V617F-positive PV patients and its influence on the disease-related complications.

\section{Materials and Methods}

We analyzed ninety JAK2V617F-positive PV patients diagnosed at the Department of Hematology, AIIMS, New Delhi, India, between 2012 and 2016. All patients were categorized according to the WHO 2008 criteria. The general characteristics of the patients are shown in Table 1. CMPN patients other than PV were excluded from the study. Ethical approval for the study protocol was obtained and written informed consent was taken from all the patients.

Granulocytes were separated from peripheral blood $(10 \mathrm{ml})$ and genomic DNA was extracted using conventional gradient centrifugation technique. All patients were routinely genotyped for the JAK2 V617F mutation by an allele-specific (ASO) polymerase chain reaction (PCR) exactly as described by Baxter et al. ${ }^{[4]}$ This approach allows both normal and mutant alleles to be visualized and can distinguish between homozygous and heterozygous mutations. These positive samples were further quantified for the allele burden of the JAK2V617F mutation using real-time PCR (RQ-PCR) with 7500 RT-PCR system (Applied Biosystems, Hayward, CA, USA). The RT-PCR was performed using TRUPCR JAK2V617F kit (3B BlackBio Biotech India Ltd., India). The TRUPCR JAK2V617F kit is based on standard curve method to quantify JAK2V617F allele burden. The RT-PCR mixture ( $20 \mu \mathrm{L}$ final volume) contained $10 \mu \mathrm{l}$ master mix, DNA template (total for about $100 \mathrm{ng}$ ), deionized water, $1 \mu \mathrm{l}$ primer probe mix for wild type and

This is an open access journal, and articles are distributed under the terms of the Creative Commons Attribution-NonCommercial-ShareAlike 4.0 License, which allows others to remix, tweak, and build upon the work non-commercially, as long as appropriate credit is given and the new creations are licensed under the identical terms.

For reprints contact: reprints@medknow.com

How to cite this article: Sazawal S, Singh K, Chhikara S, Chaubey R, Mahapatra M, Saxena R. Influence of JAK2V617F allele burden on clinical phenotype of polycythemia vera patients: A study from India. South Asian J Cancer 2019;8: 127-9. 
mutant allele detection. Amplification was performed by a standard protocol recommended by the manufacturer $\left(94^{\circ} \mathrm{C}\right.$ for $10 \mathrm{~min} ; 40$ repeated cycles of $94^{\circ} \mathrm{C}$ for $15 \mathrm{~s}, 60^{\circ} \mathrm{C}$ for $\left.60 \mathrm{~s}\right)$. The reaction of each sample was run in triplicates. The JAK2V617F proportion was calculated from standard curve generated with wild-type JAK2 and JAK2 V617F standards.

The JAK2V617F mutant copy numbers (CNV617F) and JAK2 wild-type copy numbers (CNwt) obtained in the test results were used to calculate the JAK2V617F allele burden for the patient samples. We analyzed the JAK2V617F allele burden of the patients by taking $>50 \%$ as the cutoff value for homozygosity and patients with allele burden $\leq 50 \%$ were taken as heterozygous.

JAK 2 V617F allele burden $\%=\frac{\mathrm{CNV} 617 \mathrm{~F}}{\mathrm{CNV} 617 \mathrm{~F}+\mathrm{CNwt}} \times 100$

\section{Statistical analysis}

Numerical variables were tested for normal distribution with the Kolmogorov-Smirnov test. Data were expressed as mean \pm standard deviation. The nonparametric Pearson's product-moment correlation analysis was used to test for the relationship between JAK2V617F allele burden and different clinical variables. All tests for statistical significance were two tailed and $P<0.05$ was considered statistically significant.

\section{Results}

Patient characteristics of all the ninety JAK2V617F-positive PV patients at diagnosis are summarized in Table 1. Of the ninety patients, $74(82.22 \%)$ were males and $16(17.78 \%)$ were females. The patients were divided into two groups based on age; 35 $(38.80 \%)$ patients were $\leq 50$ years and $55(61.20 \%)$ patients were $>50$ years. Patients with age $>50$ years had significantly higher JAK2V617F allele burden (median $40.15 \%$, range $0.49 \%-$ $91.62 \%$ ) as compared to patients with age $\leq 50$ years (median $48.59 \%$, range $0.56 \%-86.74 \% ; P<0.032$ ) [Table 1 ].

In all the patients, hemoglobin and hematocrit were $>15 \mathrm{~g} / \mathrm{dl}$ and $45 \%$, respectively. There were $17(18.89 \%)$ patients with WBC $\leq 10 \times 10^{9} / \mathrm{L}$ and $73(81.11 \%)$ patients with WBC $>10 \times 10^{9} / \mathrm{L}$ (median $17.60 \times 10^{9} / \mathrm{L}$, range: $4.7-55.4 \times 10^{9} / \mathrm{L}$ ). The patients with leukocytosis had higher JAK2V617F allele burden than the patients with no leukocytosis $(P<0.090)$, but was not statistically significant. There were 29 (32.22\%) patients with platelet count of $\leq 300 \times 10^{9} / \mathrm{L}$ and $61(67.78 \%)$ patients with platelet count $>300 \times 10^{9} / \mathrm{L}$ (median $436 \times 10^{9} / \mathrm{L}$, range: $3.3-1241 \times 10^{9} / \mathrm{L}$ ).

Spleen size was available in $52 / 90$ patients. Of the 52 patients, $31(59.62 \%)$ had splenomegaly at the time of diagnosis and $21(40.38 \%)$ were negative for splenomegaly. Patients with splenomegaly had significantly higher JAK2V617F allele burden (mean 50.24\%, range: $6.91 \%-84.17 \%$ ) as compared to patients without splenomegaly (mean $33.82 \%$, range: $0.49 \%-71.83 \% ; P<0.017$ ). History of thrombosis was available in $45 / 90$ patients. Ten $(22.44 \%)$ patients had been recorded for thrombotic events and $35(77.56 \%)$ patients had absence of thrombosis. Patients with higher allele burden (median 57.20, range: $43.4 \%-72.03 \%$ ) had significantly raised thrombotic events as compared to patients with lower allele burden (median 37.38, range: $49 \%-84.17 \% ; P<0.043$ ) [Table 1 ].

Out of the ninety patients, 49 were homozygous and 41 were heterozygous by ASO-PCR. A total of 32/49 (66\%) patients had allele burden $>50 \%$ which was confirmed by RT-PCR. However, 17/49 (34\%) patients who were homozygous by ASO-PCR had allele burden $<50 \%$ which may be due to mixed population of wild-type allele along with the mutant allele, thus leading to reduction of allele burden $<50 \%$. The proportion of JAK2V617F allele burden is shown in Table 2 .

\section{Discussion}

The identification of JAK2 V617F mutation is included as genetic diagnostic criteria for classification of CMPN and has been a cornerstone in understanding the pathophysiology of Philadelphia-negative CMPNs. A study by Scott et al. reported that JAK2V617F mutation did not occur in nonhematological cancers and was rare in myeloid malignancies other than the classic Philadelphia chromosome-negative CMPNs. ${ }^{[18]}$ Studies have shown that in CMPN patients, the increase of JAK2V617F allele burden has a major influence on prognostic variables and clinical phenotype of the disease. ${ }^{[1,13]}$

Elevated JAK2V617F allele burden has been found to be associated with higher risk of myelofibrosis, increased hemoglobin levels, and systemic symptoms. However, its effect on WBC counts, splenomegaly, and thrombotic risk is controversial. ${ }^{[11,13]}$ Previous reports have suggested that JAK2V617F allele burden could be related to spleen size and WBC count and is quite variable in Philadelphia chromosome-negative CMPN patients, which in part contributes to the clinical course of CMPN. ${ }^{[19,20]}$

Table 1: Clinical characteristic of ninety patients with JAK2V617F mutation

\begin{tabular}{|c|c|c|c|}
\hline Variables & $n=90(\%)$ & $\begin{array}{c}\text { JAK2V617F } \\
\text { burden } \\
\text { (Median, \%) }\end{array}$ & $P$ \\
\hline \multicolumn{4}{|l|}{ Age, Years } \\
\hline$\leq 50$ & $35(38.8)$ & $40.15(.56-86.74)$ & 0.0327 \\
\hline$\geq 50$ & $55(61.1)$ & $48.59(0.49-91.62)$ & \\
\hline \multicolumn{4}{|l|}{ Gender } \\
\hline Male & $74(82.22)$ & $43.64(0.49-86.74)$ & 0.6311 \\
\hline Female & $16(17.78)$ & 34.76 (17.24-91.62) & \\
\hline Hemoglobin (g/dl) & & & - \\
\hline$\leq 15$ & $90(100)$ & $43.49(0.49-91.62)$ & \\
\hline$\geq 15$ & 0 & & \\
\hline HCT (\%) & & & - \\
\hline$\leq 45$ & 0 & $43.9(0.49-91.62)$ & \\
\hline$\geq 45$ & 90 & & \\
\hline \multicolumn{4}{|l|}{ WBC $\left(\times 10^{9} / \mathrm{L}\right)$} \\
\hline$\leq 10$ & $17(18.89)$ & $37.05(0.49-91.62)$ & 0.090 \\
\hline$\geq 10$ & $73(81.11)$ & $46.72(1.31-89.26)$ & \\
\hline \multicolumn{4}{|l|}{$\operatorname{PLT}\left(\times 10^{9} / \mathrm{L}\right)$} \\
\hline$\leq 300$ & $29(32.22)$ & $49.18(0.49-89.26)$ & 0.508 \\
\hline$\geq 300$ & $61(67.78)$ & $40.52(0.56-91.62)$ & \\
\hline Splenomegaly & & & 0.017 \\
\hline Yes & $31(52,59.62)$ & $50.43(6.91-84.17)$ & \\
\hline No & $21(52,40.38)$ & $32.4(0.49-71.83)$ & \\
\hline Thrombosis & & & 0.043 \\
\hline Yes & $10(45,22.44)$ & $57.20(43.4-72.03)$ & \\
\hline No & $35(45,77.56)$ & $37.38(0.49-84.17)$ & \\
\hline
\end{tabular}

Table 2: JAK2V617F allele burden in polycythemia vera

\begin{tabular}{lcc}
\hline Allele burden & $\boldsymbol{n}=\mathbf{9 0}(\%)$ & Mean \pm SD \\
\hline $0.1-20 \%$ & $13(14.44)$ & $8.6 \pm 7.47$ \\
$21-40 \%$ & $22(24.44)$ & $27.96 \pm 5.46$ \\
$41-60 \%$ & $31(34.44)$ & $48.76 \pm 5.46$ \\
$61-80 \%$ & $20(22.22)$ & $66.06 \pm 4.8$ \\
$81-100 \%$ & $4(4.44)$ & $87.94 \pm 3.2$ \\
\hline
\end{tabular}

South Asian Journal of Cancer $\bullet$ Volume 8 Issue 2 April-June 2019 
In this study, we investigated JAK2V617F allele burden in PV patients and correlated it with the clinical phenotype. Our study confirmed a significant correlation of the JAK2V617F allele burden with increased age, splenomegaly, and thrombosis. PV patients with age $>50$ years had significantly higher JAK2V617F allele burden when compared to patients with age $\leq 50$ years $(P<0.032)$. This is in line with a study from the West by Tefferi et al.; however, Zhao et al. did not find any effect of age in Chinese population. ${ }^{[8,21]}$

We observed that the patients with splenomegaly had significantly higher JAK2V617F allele burden as compared to patients without splenomegaly $(P<0.017)$. Our results are in concordance with the study conducted by Vannucchi et al. in Italian population and Zhao et al. in Chinese population. ${ }^{[11,21]}$ However, Tefferi et al. did not find any correlation of allele burden with splenomegaly. ${ }^{[12]}$

The impact of JAK2V617F mutation on thrombosis is reported to be controversial in MPN patients. Vannucchi et al. reported that JAK2V617F mutation had a significant association with thrombosis. ${ }^{[22]}$ However, Passamonti et al. showed nonsignificant correlation with the risk of thrombosis in Italian PV patients. ${ }^{[20]}$ In our study, we observed that patients with higher allele burden had significantly raised thrombotic events as compared to patients with lower allele burden $(P<0.043)$.

Regarding influence of the JAK2V617F allele burden on gender, studies have reported that male PV patients had higher allele burden than females. ${ }^{[5,20]}$ However, we did not find any significant correlation of gender with allele burden, which is confirmed by Zhao et al. in Chinese patients. ${ }^{[21]}$

We found that the patients with leukocytosis had higher JAK2V617F allele burden than the patients without leukocytosis $(P=0.090)$, though not statistically significant. This is in contrast to a study from the West by Tefferi et al. who found significant correlation of leukocytosis with the allele burden. ${ }^{[12]}$

\section{Conclusion}

JAK2V617F allele burden correlated with the phenotypic presentation of PV such as increased age, splenomegaly, and thrombosis. Thus, it may be considered for prognostication and setting up the treatment protocol in PV patients. Larger studies are needed to confirm these observations.

\section{Acknowledgments}

The authors wish to acknowledge Mr. Rajender Chhoker and Mr. Arun Dalsus for technical assistance.

\section{Financial support and sponsorship}

Nil.

\section{Conflicts of interest}

There are no conflicts of interest.

\section{References}

1. James C, Ugo V, Le Couédic JP, Staerk J, Delhommeau F, Lacout C, et al. A unique clonal JAK2 mutation leading to constitutive signalling causes polycythaemia Vera. Nature 2005;434:1144-8.

2. Kralovics R, Passamonti F, Buser AS, Teo SS, Tiedt R, Passweg JR, et al. A gain-of-function mutation of JAK2 in myeloproliferative disorders. N Engl J Med 2005;352:1779-90.
3. Levine RL, Wadleigh M, Cools J, Ebert BL, Wernig G, Huntly BJ, et al. Activating mutation in the tyrosine kinase JAK2 in polycythemia vera, essential thrombocythemia, and myeloid metaplasia with myelofibrosis. Cancer Cell 2005;7:387-97.

4. Baxter EJ, Scott LM, Campbell PJ, East C, Fourouclas N, Swanton S, et al. Acquired mutation of the tyrosine kinase JAK2 in human myeloproliferative disorders. Lancet 2005;365:1054-61.

5. Larsen TS, Pallisgaard N, Møller MB, Hasselbalch HC. The JAK2 V617F allele burden in essential thrombocythemia, polycythemia vera and primary myelofibrosis - Impact on disease phenotype. Eur J Haematol 2007;79:508-15.

6. Campbell PJ, Baxter EJ, Beer PA, Scott LM, Bench AJ, Huntly BJ, et al. Mutation of JAK2 in the myeloproliferative disorders: Timing, clonality studies, cytogenetic associations, and role in leukemic transformation. Blood 2006; 108:3548-55.

7. Kralovics R, Teo SS, Buser AS, Brutsche M, Tiedt R, Tichelli A, et al. Altered gene expression in myeloproliferative disorders correlates with activation of signaling by the V617F mutation of JAK2. Blood 2005; 106:3374-6.

8. Tefferi A, Sirhan S, Lasho TL, Schwager SM, Li CY, Dingli D, et al. Concomitant neutrophil JAK2 mutation screening and PRV-1 expression analysis in myeloproliferative disorders and secondary polycythaemia. Br J Haematol 2005; 131:166-71.

9. Lippert E, Boissinot M, Kralovics R, Girodon F, Dobo I, Praloran V, et al. The JAK2-V617F mutation is frequently present at diagnosis in patients with essential thrombocythemia and polycythemia vera. Blood 2006; 108: 1865-7.

10. Passamonti F, Rumi E, Pietra D, Della Porta MG, Boveri E, Pascutto C, et al. Relation between JAK2 (V617F) mutation status, granulocyte activation, and constitutive mobilization of CD34+cells into peripheral blood in myeloproliferative disorders. Blood 2006;107:3676-82.

11. Vannucchi AM, Antonioli E, Guglielmelli P, Rambaldi A, Barosi G, Marchioli R, et al. Clinical profile of homozygous JAK2 617V $>F$ mutation in patients with polycythemia vera or essential thrombocythemia. Blood 2007; 110:840-6.

12. Tefferi A, Strand JJ, Lasho TL, Knudson RA, Finke CM, Gangat N, et al. Bone marrow JAK2V617F allele burden and clinical correlates in polycythemia vera. Leukemia 2007;21:2074-5

13. Tefferi A, Lasho TL, Schwager SM, Strand JS, Elliott M, Mesa R, et al. The clinical phenotype of wild-type, heterozygous, and homozygous JAK2V617F in polycythemia vera. Cancer 2006;106:631-5.

14. Cheung B, Radia D, Pantelidis P, Yadegarfar G, Harrison C. The presence of the JAK2 V617F mutation is associated with a higher haemoglobin and increased risk of thrombosis in essential thrombocythaemia. $\mathrm{Br} J$ Haematol 2006; 132:244-5.

15. Finazzi G, Rambaldi A, Guerini V, Carobbo A, Barbui T. Risk of thrombosis in patients with essential thrombocythemia and polycythemia vera according to JAK2 V617F mutation status. Haematologica 2007;92: 135-6.

16. Sazawal S, Bajaj J, Chikkara S, Jain S, Bhargava R, Mahapatra M, et al. Prevalence of JAK2 V617F mutation in Indian patients with chronic myeloproliferative disorders. Indian J Med Res 2010;132:423-7.

17. Singh N, Sazawal S, Upadhyay A, Chhikara S, Mahapatra M, Saxena R, et al. Correlation of JAK2V617F mutational status in primary myelofibrosis with clinico-hematologic characteristics and international prognostic scoring system scoring: A single center experience. Indian J Pathol Microbiol 2015;58: 187-91.

18. Scott LM, Campbell PJ, Baxter EJ, Todd T, Stephens P, Edkins S, et al. The V617F JAK2 mutation is uncommon in cancers and in myeloid malignancies other than the classic myeloproliferative disorders. Blood 2005; 106:2920-1.

19. Barosi G, Bergamaschi G, Marchetti M, Vannucchi AM, Guglielmelli P, Antonioli $\mathrm{E}$, et al. JAK2 V617F mutational status predicts progression to large splenomegaly and leukemic transformation in primary myelofibrosis. Blood 2007;110:4030-6.

20. Passamonti F, Rumi E, Pietra D, Elena C, Boveri E, Arcaini L, et al. A prospective study of 338 patients with polycythemia vera: The impact of JAK2 (V617F) allele burden and leukocytosis on fibrotic or leukemic disease transformation and vascular complications. Leukemia 2010;24:1574-9.

21. Zhao S, Zhang X, Xu Y, Feng Y, Sheng W, Cen J, et al. Impact of JAK2V617F mutation burden on disease phenotype in Chinese patients with JAK2V617F-positive polycythemia vera (PV) and essential thrombocythemia (ET). Int J Med Sci 2016;13:85-91.

22. Vannucchi AM. JAK2 mutation and thrombosis in the myeloproliferative neoplasms. Curr Hematol Malig Rep 2010;5:22-8. 